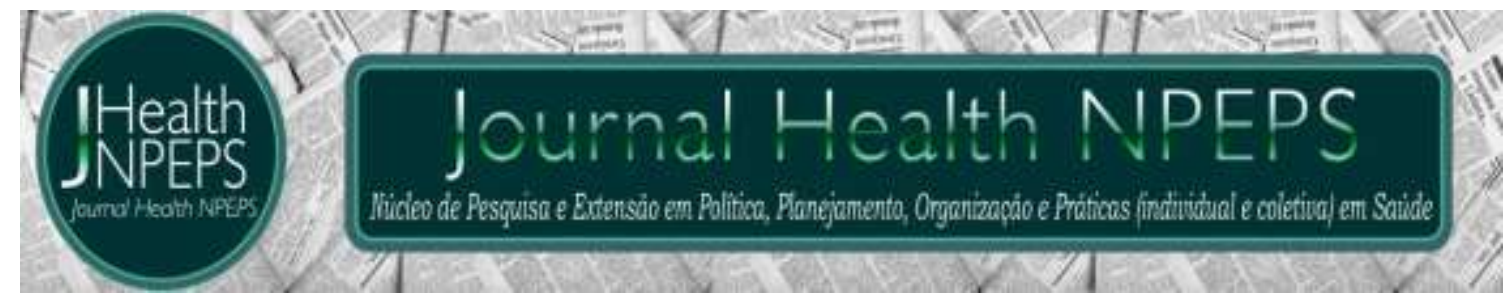

http://dx.doi.org/10.30681/252610103214

ARTIGO DE REVISÂO

\title{
Manifestações e tratamento da necrólise epidérmica tóxica e da síndrome de Stevens Johnson
}

\section{Manifestations and treatment of toxic epidermic necrolysis and Stevens Johnson's syndrome}

\section{Manifestaciones y tratamiento de la necrolisis epidérmica tóxica y del síndrome de Stevens Johnson}

\section{Cristina Ferreira Roviello ${ }^{1}$, Francisco Sandro Menezes Rodrigues ${ }^{2}$, João Antônio Bertolini Gonçalves ${ }^{3}$, Renato Ribeiro Nogueira Ferraz ${ }^{4}$}

\section{RESUMO}

Objetivo: identificar as manifestações clínicas da necrólise epidérmica tóxica (NET) e síndrome de Stevens Johnson (SSJ). Método: trata-se de uma revisão narrativa. A busca dos artigos utilizou a ferramenta Publish or Perish, que ranqueia os trabalhos com base no número de citações recebidas. Foram realizadas duas buscas, pois apesar das doenças se relacionarem, possuem diagnósticos diferentes. $\mathrm{Na}$ primeira, os descritores utilizados foram: "necrólise epidérmica tóxica" e "manifestações clínicas", e na segunda os descritores foram: "Síndrome de Stevens-Johnson" e "manifestações clínicas”. Resultados: após a leitura dos 12 artigos selecionados, entendeu-se que a patogênese da necrólise epidérmica tóxica e Síndrome de Stevens Johnson se dá pela hipersensibilidade tardia a fármacos. As manifestações clínicas se dão pelo aparecimento do eritema cutâneo com formação de

\footnotetext{
${ }^{1}$ Enfermeira. Especialista em Enfermagem Dermatológica. Universidade São Caetano do Sul (USCS). São Caetano do Sul, SP, Brasil. E-mail: crisroviello@bol.com.br ORCID ID: https://orcid.org/ 0000-0002-5923216X

${ }^{2}$ Farmacêutico. Doutor em Farmacologia. Pesquisador e Docente das Residências Multiprofissionais da Universidade Federal de São Paulo (UNIFESP). São Paulo, SP, Brasil. E-mail: sandromrodrigues@hotmail.com ORCID ID: https://orcid.org/0000-0001-7913-0585.

${ }^{3}$ Médico. Especialista em Dermatologia. Faculdade de Ciências Médicas de Minas Gerais. Instituto Superior de Medicina e Dermatologia - ISMD. Belo Horizonte, MG, Brasil. E-mail: joaoabertolini@gmail.com ORCID ID: https://orcid.org/0000-0002-6637-095.

${ }^{4}$ Biólogo. Doutor em Ciência Básicas. Docente do Programa de Mestrado Profissional em Administração - Gestão em Sistemas de Saúde (PMPA-GSS) - Universidade Nove de Julho (UNINOVE). São Paulo, SP, Brasil. E-mail: renatobio@hotmail.com ORCID ID: https://orcid.org/0000-0002-8931-895X. Autor principal - Endereço para correspondência: Rua Vergueiro, 235/239 - 12o. andar, Liberdade, CEP: 01504001, São Paulo, SP - Brasil.
}

Este artigo está licenciado sob forma de uma licença Creative Commons Atribuição 4.0 Internacional, que permite uso irrestrito, distribuição e reprodução em qualquer meio, desde que a publicação original seja corretamente citada. 
máculas, pápulas, vesículas e bolhas associadas ou isoladas, como placas de urticária ou eritema extenso. Na NET é possível notar desprendimento extenso da epiderme maior que $30 \%$ da superfície corpórea, conhecido como sinal de Nikolsky, com acometimento de mucosas. Conclusão: A NET e SSJ são farmacodermias graves, com baixas incidências, mas elevada mortalidade. 0 reconhecimento precoce das doenças e a retirada do fármaco causador são essenciais para conduzir o tratamento, diminuindo por sua vez a taxa de mortalidade.

Descritores: Enfermagem; Dermatologia; Sinais e Sintomas; Tratamento; Gestão em Saúde.

\section{ABSTRACT}

Objective: to identify the clinical manifestations of toxic epidermal necrolysis (TEN) and Stevens Johnson syndrome (SJS). Method: the articles search was done using the Publish or Perish computational tool, which ranks the articles based on the number of citations. Two separate searches were performed, because although the diseases are related, they have different diagnoses. In the first, the descriptors used were "Toxic Epidermal Necrolysis" and "clinical manifestations", and in the second the descriptors were "Stevens-Johnson Syndrome" and "clinical manifestations". Results: in total, 12 articles constituted the present revision. It was understood that the pathogenesis of TEN and SJS is due to late drugs hypersensitivity. The clinical manifestations are due to the appearance of cutaneous erythema with the formation of macules, papules, vesicles and associated or isolated blisters, such as urticaria plaques or extensive erythema. In the TEN it is possible to notice extensive detachment of the epidermis greater than $30 \%$ of the body surface, known as Nikolsky's signal, with mucous involvement. Conclusion: TEN and SJS are serious skin diseases, with low incidences but high mortality. Early recognition of disease and withdrawal of the causative drug are essential for conducting treatment, thus decreasing the mortality rate.

Descriptors: Nursing; Dermatology; Signs and Symptoms; Treatment; Health Management.

\section{RESUMEN}

Objectivo: identificar las manifestaciones clínicas de la necrólisis epidérmica tóxica (NET) y el síndrome de Stevens Johnson (SSJ). Método: la selección de los artículos consideró el número de citas recibidas por otras publicaciones. Se realizaron dos búsquedas, pues a pesar de las enfermedades se relacionan, poseen diferentes diagnósticos. En la primera, los descriptores utilizados fueron: "Toxic Epidermal Necrolysis" y "clinical manifestations", y en los segundos los descriptores fueron: "Stevens-Johnson Syndrome" y "clinical manifestations". Resultados: en total, 12 artículos constituyeron la presente revisión. Se ha comprobado que la patogénesis de TEN y SJS se debe a las drogas de larga duración. Las manifestaciones clínicas se deben a la apariencia de cutánea erythema con la formación de macules, papules, vesicles y asociados, o blister, tales como urticaria plaquetas o extensión erythema. En el TEN es posible que tenga un detalle detallado de las epidermis mayor que el $30 \%$ de la superficie del cuerpo, conocidas la Nikolsky de la señal, con mucous. Conclusión: la NET y SSJ son farmacodermias graves, con bajas incidencias pero elevada mortalidad. El reconocimiento 
precoz de las enfermedades y la retirada del fármaco causante son esenciales para conducir el tratamiento, disminuyendo a su vez la tasa de mortalidad.

Descriptores: Enfermería; Dermatología; Signos y Síntomas; Tratamiento; Gestión en Salud.

\section{INTRODUÇÃO}

A pele é considerada o maior órgão do corpo humano composto por duas camadas, cada uma com sua importância e propriedade: a epiderme camada externa da pele, e a derme, logo abaixo, com feixes de colágeno responsáveis pela sustentação de terminações nervosas e vasos sanguíneos. Abaixo da pele se encontra o tecido subcutâneo, também conhecido como hipoderme, e composto por tecido adiposo e tecido conjuntivo frouxo ${ }^{1}$. A pele age como barreira de proteção contra agressões oriundas do meio externo, como por exemplo, traumas, produtos químicos e/ou biológicos, dentre outros $^{1,2}$.

\section{A Necrólise Epidérmica} Tóxica (NET) e a Síndrome de Stevens Johnson (SSJ) são consideradas farmacodermias graves, causadas na maioria das vezes pelo uso de fármacos, embora possam existir outros fatores desencadeadores. São caracterizadas pela alta taxa de morbidade e mortalidade, e acometem a pele e a membrana mucosa, requerendo cuidados de medicina intensiva 1.

A NET e SSJ são consideradas doenças graves, gerando ruptura abrupta na pele e mucosa, impedindo a hemostasia e deixando, na maioria das vezes, sequelas irreversíveis tanto físicas como emocionais, o que gera a necessidade de um olhar e cuidados diferenciados de uma equipe multidisciplinar ${ }^{1-6}$.

Em 1922, Stevens e Johnson relataram dois pacientes com erupções cutâneas generalizadas, febre contínua, mucosa oral inflamada e conjuntiva purulenta grave, caracterizado como Eritema Multiforme Major (EMM). A partir de 1983, tais características passaram a ser conhecida como $\mathrm{SSJ}^{5,6}$.

Em 1956, A. Lyell descreveu quatro casos de pacientes com erupção da pele em forma de escaldo, como na queimadura, causada por toxicidade de medicamentos e caracterizando 
assim a NET. Esta apresenta ainda os mesmos sintomas, associados ou não aos sintomas da SSJ, mas com acometimento de área do corpo muito maior, sendo sua característica principal a necrose da epiderme em grande parte do corpo, o que requer o mesmo tratamento de um grande queimado $^{2-5}$.

A principal causa da NET e SSJ é o uso de fármacos. Consideradas reações adversas graves a medicamentos, caracterizam problema de saúde pública, enfatizando a necessidade de notificação para melhor investigação da farmacocinética e farmacovigilância ${ }^{2,4,6}$.

Perante a escassez de informações sobre essas enfermidades, o estudo objetivou identificar as manifestações clínicas da necrólise epidérmica tóxica e síndrome de Stevens Johnson, a fim de instrumentalizar os profissionais para o diagnóstico precoce.

\section{MÉTODO}

Trata-se de uma revisão narrativa, adotando como método de pesquisa a prática baseada em evidências (PBE), colaborando para inserção de evidências científica na prática clínica. Permite reunir e sintetizar de maneira sistemática os resultados da pesquisa sobre 0 tema abordado ${ }^{1}$.

A busca dos artigos foi realizada no mês de outubro de 2018 por meio da ferramenta computacional Publish or Perish, que seleciona os trabalhos com base no número de citações recebidas por outros trabalhos.

Foram realizadas duas buscas separadas, visto que apesar das doenças se relacionarem, possuem diagnóstico diferentes. $\mathrm{Na}$ primeira busca os descritores utilizados foram: necrólise epidérmica tóxica e "manifestações clínicas. Na segunda busca os descritores utilizados foram: síndrome Stevens-Johnson e manifestações clínicas.

Foram respeitados todos os aspectos éticos envolvendo esse caráter investigativo, sinalizando e informando todas as fontes de dados utilizadas.

\section{RESULTADOS}


$\mathrm{Na}$ primeira busca foram identificados 998 trabalhos com 6.244 citações, e na segunda 980 trabalhos com 10.468 citações. Foram incluídos artigos originais, artigos de revisão e estudos de caso, disponíveis na íntegra e em português ou espanhol, sem a observação de corte temporal. Foram excluídos artigos em inglês e que não possuíam o índice $\mathrm{H}$. A exclusão dos artigos em inglês visou identificar apenas os trabalhos nacionais e de países latinos.

Após a leitura inicial dos títulos e resumos dos trabalhos previamente identificados, 12 artigos respeitaram os critérios de inclusão previamente estabelecidos, estes foram lidos na íntegra e apresentados de forma compilada.

\section{REVISÃO DA LITERATURA}

Epidemiologia e manifestações clínicas da NET e SSJ

A epidemiologia da NET e SSJ se definem como patologias raras com incidência estimada entre 1 a 3 casos por milhão de habitantes por ano na população geral. A NET acomete 0,4 a 1,2 milhões de pessoas por ano, com 30\% de mortalidade. Já a SSJ atinge entre 1,2 a 6 por milhões de indivíduos ao ano, com $5 \%$ de taxa de mortalidade. Alguns fatores podem contribuir para o aumento da morbidade e mortalidade dessas doenças, como doenças com ativação imune, doença crônica e idade avançada ${ }^{6-10}$.

A patogênese se dá por uma hipersensibilidade tardia a fármacos. A imunoglobulina (lgG) deposita-se na junção dermoepidérmica e nos pequenos vasos da derme. 0 antígeno leucocitário humano (HLA-DR) é ativado, ocorrendo uma desordem nos queratinócitos com subsequente resposta inflamatória na pele. As células T CD8+ reconhecem 0 complexo maior de histocompatibilidade I (MHC-I), modificadas por um antígeno responsável por produzirem as lesões na NET e SSJ (8). Em 50\% dos casos existe uma deficiência da enzima glutationa transferase, que pode estar relacionada à SSJ. $\mathrm{Na}$ histopatologia verifica-se necrose em células satélites nos estágios iniciais, evoluindo para necrose epidérmica e inflamação da derme. 
A NET apresenta uma inflamação da derme menor, mas com maior destruição epidérmica ${ }^{6,7,11,12}$.

A etiologia da NET e SSJ, em $80 \%$ dos casos, se dá por uma hipersensibilidade causada por alguns fármacos. Porém, existem situações onde o uso do fármaco não está relacionada à manifestação da doença, que podem ser ativadas em $20 \%$ dos casos por agentes infecciosos tais como vírus, bactérias, fungos e protozoários, ou mesmo causadas por tumores, ${ }^{6,8,12}$. Os principais agentes etiológicos envolvidos são apresentados no Quadro 1.

Quadro 1 - Principais agentes etiológicos envolvidos na NET e SSJ.

\begin{tabular}{|l|l|}
\hline Medicações: & $\begin{array}{l}\text { Sulfatdiazina, } \\
\text { sulfametoxazol- } \\
\text { trimetoprim, fenobarbital, } \\
\text { fenitoína, carbamazepina, } \\
\text { alopurinol, penicilinas, } \\
\text { sulfassalazina, } \\
\text { fenilbutazona, lamotrigina }\end{array}$ \\
\hline Virais: & $\begin{array}{l}\text { Vírus Herpes Simples } \\
\text { (HSV), HIV, Coxsackie, } \\
\text { Influenza, Hepatite, } \\
\text { Varíola, Entereviroses, } \\
\text { Epstein Bar Vírus }\end{array}$ \\
\hline Bactérias: & $\begin{array}{l}\text { Estreptococo Beta } \\
\text { Hemolítico do grupo A, } \\
\text { Difteria, Brucelose, } \\
\text { Micobactérias, } \\
\text { Micoplasma, Tularemia e } \\
\text { Febre Tifoide }\end{array}$ \\
\hline Fúngicos: & $\begin{array}{l}\text { Paracocidiodomicose, } \\
\text { Dermatofitoses } \\
\text { Histoplasmose }\end{array}$ \\
\hline Protozoários: & Malária e Tricomonas \\
\hline Tumores: & Carcinomas e Linfomas \\
\hline
\end{tabular}

Fonte: Nagão-Dias ${ }^{6}$, Chávez-Lemus ${ }^{5}$, Bulisani $^{8}$, Oliveira ${ }^{11}$ e Emerick ${ }^{12}$.
As manifestações clínicas se iniciam com a presença de eritema cutâneo, com formação de máculas que se tornam pápulas, vesículas e bolhas, associadas ou isoladas, além de placas de urticária ou eritema extenso. 0 quadro clínico característico da SSJ, no exame físico inicial, pode apresentar febre, taquicardia e hipotensão. Alteração do nível de consciência pode estar presente, e na NET é possível ainda notar desprendimento da pele e perda total da epiderme, condição conhecida como sinal de Nikolsky, deixando o paciente sem nenhuma proteção e suscetível a várias infecções, podendo inclusive evoluir para sepse, que por sua vez é maior causa de morte no paciente com NET e SSJ2,4,8,12.

$$
\text { A face, o pescoço e o tórax }
$$
são as partes do corpo mais afetadas, mas as lesões podem se estender para todo o corpo em formato de eritema, edema, ulceração e necrose, acometendo as mucosas ocular, oral, vaginal, e não raras aderências gastrintestinais $^{2-4,12}$. 
O diagnóstico é realizado por meio dos achados clínicos e suas manifestações. Não existe nenhum teste laboratorial que identifique o fármaco que causou a doença. Os testes de provocação não são indicados pois a exposição ao agente pode gerar outra SSJ ou NET. Os achados laboratoriais mais comuns são leucocitose, aumento dos marcadores de necrose tumoral e aumento da proteína $C$ reativa $(P C R)^{5,8,9,11}$. A biópsia da pele é realizada como teste complementar, geralmente sendo identificada presença de linfócitos T-CD4, e necrose epidérmica com metaplasia epitelial escamosa, além de eosinofilia 2,7,8,12.

Existe um escore utilizado para determinar critérios de gravidade da NET e SSJ, conhecido como SCORTEN, elaborado por Bastuji-Garin, que mensura 0 prognóstico e o risco de morte para paciente diagnosticados com NET e SSJ4,7,9-12, representados no Quadro 2.

O tratamento se dá a partir da identificação da reação e retirada do fármaco causador, visto que quanto mais rápido $\mathrm{o}$ reconhecimento melhor prognóstico o paciente terá. É importante que esse paciente seja assistido em uma unidade terapia intensiva, visto que alguns estudos demonstraram que a admissão em unidade de tratamento de queimados tem sido a melhor opção, já que na NET o tratamento é realizado de maneira semelhante $2,7,11,12$.

Quadro 2 - Escore de SCORTEN.

\begin{tabular}{|c|c|}
\hline $\begin{array}{l}\text { Fatores de } \\
\text { risco: }\end{array}$ & $\begin{array}{l}\text { Idade maior que } 40 \text { anos } \\
\text { Neoplasia } \\
\text { Frequência Cardíaca } \\
\text { maior que } 120 \mathrm{bpm} \\
\text { Acometimento } \\
\text { epiderme maior que } 10 \% \\
\text { Ureia maior } 28 \mathrm{mg} / \mathrm{Dl} \\
\text { Glicose sérica maior que } \\
252 \mathrm{mg} / \mathrm{Dl} \\
\text { Bicarbonato menor que } \\
20 \mathrm{mg} / \mathrm{Dl}\end{array}$ \\
\hline $\begin{array}{l}\text { Taxa de } \\
\text { mortalidade: }\end{array}$ & $\begin{array}{l}\text { SCORTEN } 0-1=3,2 \% \\
\text { SCORTEN } 2=12,1 \% \\
\text { SCORTEN } 3=35,3 \% \\
\text { SCORTEN } 4=58,3 \% \\
\text { SCORTEN } 5 \text { OU MAIS } \\
=90 \%\end{array}$ \\
\hline
\end{tabular}

O tratamento se baseia na sintomatologia, sendo oferecido de imediato cuidados especiais com as vias aéreas, controle da temperatura, que deve variar entre 30 a $32^{\circ} \mathrm{C}$, com o objetivo de prevenir perdas calóricas. Para isso podem ser utilizadas lâmpadas infravermelhas e camas ar-arfluidized, manipulação asséptica e analgesia adequada, sendo recomendado uso de opioides de maneira responsável e segura, além 
do uso de ansiolíticos. O acesso venoso deve ser preferencialmente periférico e o mais longe possível da área afetada. Deve-se evitar cateteres centrais, indicados somente em casos de uso de drogas vasoativas ou por impossibilidade de acesso venoso periférico. A reposição de eletrólitos, hidratação pele, início imediato de protetores gástricos para prevenção de ulcera gástrica por estresse, e anticoagulação para prevenção trombose venosa profunda também são de considerável importância ${ }^{7,11,12}$.

As lesões em mucosas merecem uma atenção diferenciada, e nas lesões oculares - uso de colírios e solução fisiológica a $0,9 \%$ devem ser utilizadas no intervalo em média de 3 em 3 horas, havendo a possibilidade e necessidade de antibiótico tópico, inclusive na mucosite oral, além da hidratação oral precoce $3,4,9,10,12$.

Não existe um consenso sobre as coberturas tópicas para o tratamento da pele, podendo variar da simples limpeza da pele com solução fisiológica e hidratação com óleos a base de ácidos graxos essenciais, até a utilização de coberturas mais modernas como o hidroalginato com prata. Este, por sua vez, já demonstrou uma resposta mais rápida de cicatrização, mas com custo muito mais elevado, não estando disponível em todos os serviços de saúde. Também não se deve desconsiderar o desbridamento em centro cirúrgico nos casos da $\mathrm{NET}^{7,12}$. A inspeção cuidadosa e diária é necessária para prevenir possíveis complicações e infecções secundárias, como por exemplo, no trato respiratório e urinário, sendo necessária a introdução de antibióticos sistêmicos, que são indicados somente nesses casos, já que uso profilático pode causar resistência bacteriana ${ }^{5,9,11,12}$.

Ainda para o tratamento são utilizados alguns fármacos, como as imunoglobulinas, ciclosporina, ciclosfosfamida, pentoxifilina e a talidomida, que ainda está em discussão sobre seus benefícios frente à NET e a SSJ. Os esteroides não são indicados por aumentar o risco de infecções e não encurtarem 0 tempo de tratamento das doenças, apesar de alguns estudos indicarem que o uso 
da pulsoterapia no início do diagnóstico é controversa em relação às contraindicações dos corticoides $^{7,12}$.

Indica-se na NET e SSJ a coleta e preparação de culturas a cada 48 horas, que deve ser realizada na pele, sangue, urina e mucosa, além de comparada com culturas anteriores, guiando $\mathrm{o}$ tratamento a ser seguido. As lesões da pele dificilmente deixam cicatrizes, ao contrário das mucosites, que podem gerar complicações tardias como o estreitamento e sangramento das mucosas $^{6,8,9,12}$.

\section{CONCLUSÃO}

A NET e SSJ são farmacodermias graves, consideradas problemas de saúde pública, com baixas incidências, mas elevada mortalidade. As principais manifestações clínicas dessas doenças são o eritema cutâneo com formação de máculas, pápulas, vesículas e bolhas associadas ou isoladas, como placas de urticária ou eritema. 0 extenso quadro clínico característico da SSJ, no exame físico inicial, pode apresentar febre, taquicardia, hipotensão e alteração do nível de consciência.

$\mathrm{Na}$ NET é possível notar ainda desprendimento extenso da epiderme, conhecido como sinal de Nikolsky. 0 reconhecimento precoce das doenças e a retirada do fármaco causador são essenciais para conduzir o tratamento, diminuindo por sua vez a taxa de mortalidade.

Dentre as limitações deste estudo, destacou a não inclusão de artigos em inglês, embora que proposital, e a não inclusão na estratégia de busca de alguma palavra-chave que pudesse identificar outros artigos sobre a temática escolhida para a presente pesquisa. Nesse sentido, sugere-se a condução de estudos futuros com apreciação e aprovação prévia da estratégia de busca por um especialista nas doenças investigadas.

\section{REFERÊNCIAS}

1. Santos NDM, Ribeiro JLA, Watanabe EAMT, Bergamaschi FPR. Diagnóstico de enfermagem evidenciados em 
pacientes com feridas: uma revisão integrativa. Anais dos Eventos da enfermagem UEMS. 2017; 1(1):8-14.

2. Martínez-Cabriales

SA.

Actualidades

en

farmacodermias

severas:

síndrome de Stevens-Johnson

(SSJ) y necrólisis epidérmica tóxica (NET). Gac Med Mex. 2015; 151:777-87.

3. Brinca A, Andrade $\mathrm{P}$, Xavier MM, Gonçalo M, Figueiredo A. Síndrome de Stevens-Johnson e Necrólise Epidérmica Tóxicacasuística de 10 anos. Revista SPDV. 2011; 69(3):403.

4. Calistru AM, Lisboa C, Cunha AP, Azevedo F. Toxidermiasestudo dos casos internados num hospital central (20002010). Revista SPDV. 2011; 69(4):585.

5. Chávez-Lemus FA, Reynoso-von Drateln C, Rodríguez-Martínez N. Prevalencia de las farmacodermias en pacientes hospitalizados en el Servicio de Dermatología de la UMAE del Centro Médico Nacional de Occidente. Periodo 2004-2012. Dermatol Rev Mex. 2014; 58(4):339-45.
6. Nagao-Dias AT, Barros-Nunes P, Coelho HL, Solé D. Reações alérgicas a medicamentos. J Pediatr. 2004; 80(4):259-66.

7. Antunes J, Lestre S, Gonçalves A, Borges A, Viseu R, Baptista I. Necrólise epidérmica tóxicaValproato de sódio e vancomicina? Rev Port Imunoalergologia. 2011; 19(2):101-10.

8. Bulisani ACP, Sanches GD, Guimarães HP, Lopes RD, Vendrame LS, Lopes AC. Síndrome de Stevens-Johnson e necrólise epidérmica tóxica em medicina intensiva. Rev Bras Ter Intensiva. 2006; 18(3):2927.

9. Arguijo MB, Díaz GB, Gallego RB, Francés EC, García VC, Torrijos LT, et al. Cuidados enfermeros en paciente geriátrico con síndrome de Stevens-Johnson relacionado con levofloxacino: a propósito de un caso. Gerokomos. 2010; 21(3):118-122.

10. Monge RB, Morales KE. Sindrome de Stevens-Johnson y necrólisis epidérmica tóxica. Rev Med Costa Rica \& 
Controamerica.

2014;

71(610):305-8.

11. Oliveira FL, Silveira LK, Morais TS, Serra MCVF. Necrólise epidérmica tóxica e síndrome de Stevens Johnson: atualização. Rev Bras Queimaduras. 2012; 11(1):2630.

12. Emerick MFB, Rodrigues MMT, Pedrosa DMAS, Novaes MRCG, Gottems LBD. Síndrome de
Stevens-Johnson e Necrólise Epidérmica Tóxica em um hospital do Distrito Federal. Rev Bras Enferm. 2014; 67(6):898-904.

Conflito de interesses: Os autores declaram não haver conflito de interesses.

Participação dos autores:

- Concepção: Roviello CF, Rodrigues FSM, Gonçalves JAB, Ferraz RRN.

- Desenvolvimento: Roviello CF, Rodrigues FSM, Gonçalves JAB, Ferraz RRN.

- Redação e revisão: Roviello CF, Rodrigues FSM, Gonçalves JAB, Ferraz RRN.

Como citar este artigo: Roviello CF, Rodrigues FSM, Gonçalves JAB, Ferraz RRN. Manifestações e tratamento da necrólise epidérmica tóxica e da síndrome de Stevens Johnson. J Health NPEPS. 2019; 4(1):319-329.

Submissão: $24 / 11 / 2018$

Aceito: $02 / 04 / 2019$

Publicado: 01/06/2019 